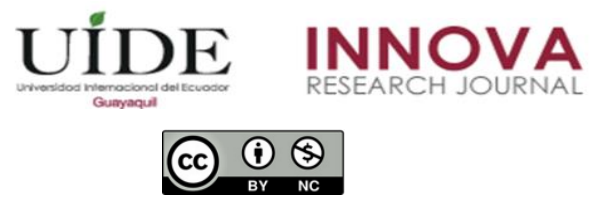

INNOVA Research Journal, ISSN 2477-9024

(Septiembre-Diciembre 2019). Vol. 4, No.3.2 pp. 205-216

DOI: https://doi.org/10.33890/innova.v4.n3.2.2019.1100

URL: http://revistas.uide.edu.ec/index.php/innova/index

Correo: innova@uide.edu.ec

\title{
Impacto de las importaciones no petroleras desde China, en la balanza comercial del Ecuador, período 2013-2017
}

\section{Impact of non-oil imports from China, in the trade balance of Ecuador, 2013- 2017 period}

\author{
Ruth Maryury Delgado Olaya \\ http://orcid.org/0000-0002-6067-9006 \\ Mariana Marisol Yánez Sarmiento \\ Universidad Metropolitana del Ecuador, Ecuador
}

Autor para correspondencia: ruthdeleg77@gmail.com; marisolyanezsami@gmail.com

Fecha de recepción: 19 de julio del 2019 - Fecha de aceptación: 13 de diciembre del 2019

\section{Resumen}

Las importaciones no petroleras ecuatorianas desde China en los últimos años, han tenido un efecto significativo en la balanza comercial, generando conocimiento sobre la situación del Ecuador durante el período analizado, en aspectos macroeconómicos como el saldo comercial que afectó al producto interno bruto (PIB) en un promedio del 3\%; las importaciones desde China han provocado una salida de capitales de 15.262 millones de dólares; provocando inestabilidad macroeconómica, entre otros. La importante cantidad de información disponible al respecto, que constituye una fuente de información secundaria, que permitió realizar un análisis comparativo percentil de las importaciones y exportaciones no petroleras de los datos recopilados. Este análisis permitió realizar ciertas conclusiones a priori, por ejemplo que para generar desarrollo y crecimiento en el Ecuador se debe apoyar a la producción y a la industrialización, generar estrategias como convenios y acuerdos bilaterales de cooperación y apoyo mutuo que favorezcan a las partes; abriendo mercados en otras latitudes, que contribuyan a mejorar las cuentas nacionales y un posible equilibrio comercial con China.

Palabras clave: importaciones; exportaciones; balanza comercial; comercio con China

\begin{abstract}
Ecuadorian non-oil imports from China in recent years have had a significant effect on the trade balance, generating knowledge about Ecuador's situation during the period analyzed, in macroeconomic aspects such as the trade balance that affected the gross domestic product (GDP) on an average of 3\%; imports from China have resulted in capital outflows of \$ 15,262 million; causing macroeconomic instability, among others. The significant amount of information available in this regard, which constitutes a secondary source of information, which allowed a comparative percentile analysis of non-oil imports and exports of the data collected. This analysis allowed certain conclusions to be drawn up a priori, for example, that in order to generate development and growth in Ecuador, production and industrialization must be supported, generating strategies such as bilateral cooperation and mutual support agreements and agreements that favor the parties;
\end{abstract}


opening markets in other latitudes, which contribute to improving national accounts and a possible trade balance with China.

Key words: imports; exports; commercial balance; impact

\section{Introducción}

Entre las tareas de todo Estado está la de implementar acciones y estrategias que apunten a mantener el equilibrio entre la oferta y demanda en los mercados nacionales e internacionales; además, el posicionamiento en el comercio internacional, ganando poder y control en la economía; para tal efecto, recurre a acuerdos, tratados y convenios sean estos bilaterales o de bloque, que buscan alternativas que beneficien a las partes.

El comercio en el contexto internacional facilita el ingreso de los productos nacionales en el mercado mundial, atrayendo a la inversión extranjera directa; uno de los ejes principales para el crecimiento y desarrollo económico de un país, atrayendo capitales para crear nuevas empresas y por ende nuevas fuentes de empleo; dinamizando la economía.

China es un ejemplo en que los acuerdos internacionales generan desarrollo y crecimiento; al posicionarse como la primera potencia mundial; representando un mercado importante para sus exportaciones, en especial de sus productos y un potencial competidor en el comercio internacional.

Por su economía, China está en constante crecimiento, se ha convertido en un socio estratégico comercial para América Latina; a través de la inversión, y préstamos bilaterales. En este sentido; las relaciones comerciales entre Ecuador y China, han obtenido un gran desarrollo, empujado aún más por los convenios bilaterales (Perrotti, 2015).

Para fundamentar lo antes expuesto, Mazo (2014) indicó que al convertirse China en la primera potencia mundial desde el año 2014; y, desplazando a Estados Unidos, que desde 1872 ocupó el primer lugar como potencia mundial; los tigres asiáticos adelantaron su crecimiento cinco años de anticipación; pronosticado para el 2019; esto debido a su rápido crecimiento en el mundo; para Zurita (2016) en el año 2000, la producción de China se había cuadruplicado; en 2010 China se convirtió en el exportador número uno del mundo; en 2011 China superó a Japón como la segunda economía del mundo y en octubre de 2014, se adelantó a los Estados Unidos y se convirtió en la primera potencia económica mundial.

Estos resultados han sido posible gracias a que China representa el poder industrial mundial número uno, el mayor exportador e importador de bienes y el mayor consumidor de productos de lujo (Zurita, 2016).

¿Por qué importar de China? Para Mora (2011) se debe importar por 2 factores:

- El bajo costo de la manufactura; y,

- La eficiencia en la línea de producción. 
Situación que pone al Ecuador en un problema macroeconómico; ya que la balanza comercial no petrolera con los países asiáticos se ubicó en el 2007 en -USD 982 millones de dólares a septiembre del 2017 se ubicó en -USD 2.1626 millones de dólares; por lo cual a partir del 2015 se establecen nuevos acuerdos bilaterales con el gigante asiático (Banco Central del Ecuador, 2018), representando millones de dólares en déficit; generando inequidad entre el comercio internacional entre ambos países. China como primera potencia mundial en comercio exterior desde 2014 y primer país donde Ecuador realiza importaciones no petroleras; ha generado un impacto negativo en la economía ecuatoriana.

Por los antecedentes señalados la presente investigación tiene como objetivo describir el impacto de las importaciones no petroleras desde China, en la balanza comercial del Ecuador, durante el período 2013-2017. Objetivos que se enfocan en determinar el saldo de la balanza comercial relacionado con importaciones y exportaciones no petroleros; la dependencia de Ecuador del mercado chino y calcular el porcentaje de exportaciones no petroleras con relación a las importaciones no petroleras entre Ecuador y China en el período 2013-2017.

Por el estudio realizado, tiene un diseño no experimental de tipo seccional descriptivo; ya que Hernández, Fernández \& Baptista (2010) explican que este tipo de diseños buscan especificar propiedades, características y rasgos importantes de cualquier fenómeno que se analice; es decir, describe tendencias de un grupo o población. Su aplicación permite describir el impacto en la balanza comercial del Ecuador como consecuencia de las importaciones no petroleras desde China en el período 2013-2017.

Además por el diseño no existe manipulación de las variables inmersas en este contexto, limitándonos a observar estos hechos tal como se dan en su contexto natural; para luego procesar la información en un análisis comparativo percentil y la interpretación correspondientes. El desarrollo de la temática planteada contribuirá a generar conocimiento en aspectos económicos sobre la situación del Ecuador durante el período analizado en relación a la balanza comercial del Ecuador con China.

\section{Importaciones del Ecuador desde China}

Las importaciones constituyen relaciones que se generan entre diferentes países para intercambiar bienes y/o servicios que el uno o el otro no poseen en la demandan de las sociedades para la satisfacción de sus necesidades locales.

De acuerdo al estudio realizado por los autores Durán \& Pellandra (2017) las importaciones ecuatorianas de productos chinos, desde 2012 se han acelerado considerablemente, al igual que en otros países de la región, especialmente en las textiles en 14,6\%, en electrónica, maquinarias el 11,7\% y equipos el 11,3\%. Es por ello que, en el 2015 Ecuador implementa salvaguardias a los productos importados tales como: prendas de vestir, preparaciones alimenticias, frutas (manzanas, uvas, peras, duraznos, entre otros), vehículos de cargas, impresoras, imprentas, muebles, calzados, manufacturas de plásticos, televisores CKD, teléfonos y sus partes (no incluyen celulares); esto se atribuye que el Ecuador no posee industrias de alto valor agregado en la producción (Feijoó, 2019). 


\section{Exportaciones hacia China}

El principal producto de exportación en el Ecuador hacia China es el petróleo, cuyo valor representa el 77\% del total de las exportaciones realizadas durante el 2010. En cambio entre los principales productos no petroleros que Ecuador exporta hacia China están: madera y manufacturas, productos de cobre y desechos, harina de pescado, camarones, desechos de aluminio, desechos de plástico, banano, frutas, botones de plástico o tagua (Peña, 2019).

De acuerdo a la oficina comercial de Ecuador en China, los productos ecuatorianos con mayor demanda en el mercado Chino son: harina y pellets, pescado, crustáceos, moluscos, madera, bananos, camarones, langostinos, aguas y glicerinas, café, pasta de cacao, café sin tostar, frutas preparadas y en conservas, chocolates y licor (La Hora, 2011).

\section{Balanza Comercial}

La balanza comercial es el saldo que surge entre el valor de las exportaciones y las importaciones; es decir, cuando las importaciones son mayores a las exportaciones, entonces se genera un saldo comercial o una balanza comercial negativa; por el contrario, si las exportaciones de un país son mayores a las importaciones se obtiene un saldo comercial positivo; por ello la balanza comercial pertenece a las cuentas corrientes, las mismas que posee distintas variables y se configuran de diferente forma:

Balanza de bienes: En este grupo se encuentran las actividades de comercio de mercancías; es decir, las importaciones y exportaciones de bienes.

Balanza de renta: Este rubro está conformado por los pagos e ingresos realizados, principalmente las rentas de inversión y la remuneración que se dan a los empleados de una determinada organización (Briones, 2018).

Para medir la balanza comercial se realiza la siguiente ecuación:

Balanza comercial: Exportaciones-Importaciones=Balanza positiva o negativa

\section{Balanza Comercial entre Ecuador y China}

A partir del año 2015 China desplazó a Estados Unidos al atribuirse el país con mayor exportaciones hacia el Ecuador; cuyas inversiones ascendieron, en primer lugar en $\$ 3.120$ millones de dólares, en segundo lugar Estados Unidos con \$ 2.583 millones de dólares y en tercer lugar Colombia con \$ 1.388 millones de dólares; aunque en el 2016 estos valores disminuyeron; para el año 2017 las estadísticas aumentaron, manteniendo el liderazgo; esto se debe, según la Revista Líderes (2018) a que China empezó a convertirse en la primera economía del planeta desplazando a Estados Unidos; a través, del lanzamiento de la campaña que se conoce como el "Sueño Chino" dando paso al continuismo de un sistema económico de desarrollo e incremento de necesidades materiales del país; y, con la visión de convertirlo en una nación moderadamente acomodada hasta el 2021 y plenamente desarrollada hasta el 2049 (Ramírez, 2014). 


\section{Tabla 1.}

Importaciones no petroleras del Ecuador de los tres principales países de origen en millones de dólares (FOB) enero - noviembre

\begin{tabular}{llllll}
\hline Importaciones & $\mathbf{2 0 1 3}$ & $\mathbf{2 0 1 4}$ & $\mathbf{2 0 1 5}$ & $\mathbf{2 0 1 6}$ & $\mathbf{2 0 1 7}$ \\
\hline China & 3917 & 3915 & 3585 & 2618 & 3141 \\
Estados Unidos & 3124 & 2938 & 2299 & 1626 & 1810 \\
Colombia & 1899 & 1809 & 1428 & 1136 & 1362 \\
\hline \multicolumn{7}{l}{ Fuente: Ministerio de Comercio Exterior del Ecuador } & $(2017)$
\end{tabular}

Ecuador es un ejemplo donde la estrategia del "Sueño Chino" representado por Xi Jinping ha dado resultados positivos para China; como se observa en la tabla 1, el gigante asiático se encuentra en la línea de lograr su objetivo mediante su programa de gobierno; Ramírez (2014) de devolverle a China su posición de primacía mundial sin olvidar el mejoramiento de la calidad de vida de los ciudadanos.

Asimismo, se observa que las importaciones que el Ecuador ha realizado desde China durante el 2013 al 2017 son de 17.176 millones de dólares; es decir, corresponde a salida de capital hacia el país asiático.

\section{Tabla 2}

Exportaciones no petroleras desde Ecuador a China en millones de dólares Americanos (FOB) enero-noviembre

\begin{tabular}{llllll} 
Exportaciones & $\mathbf{2 0 1 3}$ & $\mathbf{2 0 1 4}$ & $\mathbf{2 0 1 5}$ & $\mathbf{2 0 1 6}$ & $\mathbf{2 0 1 7}$ \\
\hline China & 234 & 391 & 518 & 383 & 388 \\
\hline \multicolumn{7}{l}{ Fuente: Ministerio de Comercio Exterior e Inversión (2017) }
\end{tabular}

"La presencia de China en la economía ecuatoriana se ve reflejada en sus múltiples participaciones en proyectos y obras civiles, inversiones financieras tanto privadas como estatales; y en actividades de explotación petrolera, por citar las más importantes" (Peña, 2018, pág. 4). Sin embargo, Ecuador y China han mantenido una relación comercial muy dispareja cuantitativamente; tal como se observa en las tablas 1 y 2 las importaciones no petroleras con relación a las exportaciones no petroleras representan cifras negativas que han llegado a dar como resultado un déficit en la balanza comercial del Ecuador que sobrepasan los millones de dólares.

Para los autores Jiménez \& otros (2017) Ecuador exporta variedad de productos hacia China como: camarones, harina de pescado, banano, productos mineros, maderas, desperdicios de metales (chatarra), concentrado de plomo y cobre, cacao, flores naturales, elaborados de banano entre otros; aun cuando el país tiene recursos atractivos para China, estos no alcanzan los niveles necesarios para mantener una economía equilibrada, que garantice la estabilidad en la balanza comercial ecuatoriana. Por el contario las importaciones no petroleras desde China, resultan para el país asiático una oportunidad de desarrollo y crecimiento. 
Asimismo, para los mismos autores Ecuador importa desde China productos como: medicamentos, sangre para uso terapéutico, instrumentos y aparatos de medicina, vehículos livianos, insecticidas, antirroedores, fungicidas, centrifugadoras, hornos industriales o de laboratorio, máquinas para preparación de alimentos y bebidas, máquinas para trabajar caucho, abonos con dos o tres elementos fertilizantes, entre otros.

\section{Tabla 3}

Relación entre exportaciones e importaciones no petroleras, Ecuador y China \% y en millones de dólares Americanos (FOB)

\begin{tabular}{llllll}
\hline Datos & $\mathbf{2 0 1 3}$ & $\mathbf{2 0 1 4}$ & $\mathbf{2 0 1 5}$ & $\mathbf{2 0 1 6}$ & $\mathbf{2 0 1 7}$ \\
\hline Importaciones & 3917 & 3915 & 3585 & 2618 & 3141 \\
Exportaciones & 234 & 391 & 518 & 383 & 388 \\
\hline Porcentaje Exp/Imp & $5,97 \%$ & $9,99 \%$ & $14,45 \%$ & $14,63 \%$ & $12,35 \%$ \\
\hline \multicolumn{5}{c}{ Fuente: Ministerio de Comercio Exterior del Ecuador $(2017)$}
\end{tabular}

De la información detallada en la tabla 3, el porcentaje de exportaciones no petroleras con relación a las importaciones no petroleras representan valores mínimos; lo que causa un déficit millonario del en la balanza comercial de 15.262 millones de dólares durante el período 2013 al 2017 en el Ecuador, demostrando así que la economía del país no suministra los bienes que sus habitantes necesitan, provocando gran inestabilidad macroeconómica.

China es un gigante industrial, con grandes ventajas competitivas y comparativas frente a Ecuador, su capacidad de producción a menor costo y el tipo de cambio de su moneda, han hecho que sus productos sean más deseables a los gustos y preferencias de los consumidores.

Con estos antecedentes es claro que las necesidades de los recursos materiales que China provee son mayores a los recursos que ofrece el Ecuador; sumado a esto, las herramientas y estrategias más importantes que el gobierno chino ha implementado para posicionarse en el mercado de América Latina como son:1) Asociaciones estratégicas integrales; que para el caso del Ecuador a partir del año 2016; 2) Foro de China-CELAC; designado a servir como carta de ruta de cinco años, para avanzar en las relaciones con China; 3) Crear un régimen comercial en el Pacífico (Ellis, 2018).

Ante las herramientas y estrategias implementadas por el gobierno chino y otros factores; el Ministerio de Comercio Exterior e Inversión (2018) informó que Ecuador se encuentra con un panorama incierto por la baja del petróleo; y, la apreciación del dólar americano frente a diferentes monedas del mundo como: el euro en (25,5\%); el peso colombiano en un (30,3\%); el sol peruano en (7,9\%); el yen japonés en (13,3\%); y el renminbi chino en $(2,7 \%)$ (De la Paz, 2018).

Para la misma autora, a los ecuatorianos les resulta más barato comprar a los países antes mencionados prendas de vestir, neumáticos o artículos de línea blanca ya que el beneficio será del 30,3\% en el caso de Colombia; en cambio en Perú el inventivo sería un promedio del 8\%; y en el caso de los países asiáticos los costos de producción son tan bajos que el beneficio ha sido por años la diferencia abismal de precios; es por este motivo, que la balanza comercial con China tema de investigación, es enormemente deficitaria para Ecuador. 
Esta situación, obligó al Ecuador a tomar medidas para regular el equilibrio de la balanza comercial imponiendo la aplicación de aranceles (salvaguardias) que van desde el 5\%; 15\%; $25 \%$ y $45 \%$ a 2.996 productos de importación para Ecuador en el año 2015; el cual tendría una duración de 15 meses.

Como resultado de la aplicación de las salvaguardias en el 2015; claramente se nota en la tabla 3 que, éstas han tenido un efecto positivo al disminuir las importaciones en el año 2016 en USD 2.618 millones de dólares americanos; lo que representa un 26,97\% de las importaciones no petroleras totales en referencia al 2015; y, para el año 2017 las importaciones no petroleras desde China aumentaron alrededor de un $19,98 \%$ con relación al año 2016; datos que se fundamentan, debido a que las salvaguardias se aplicaron desde marzo del 2015 hasta mayo 2017 como estrategia implementada por el gobierno de Ecuador.

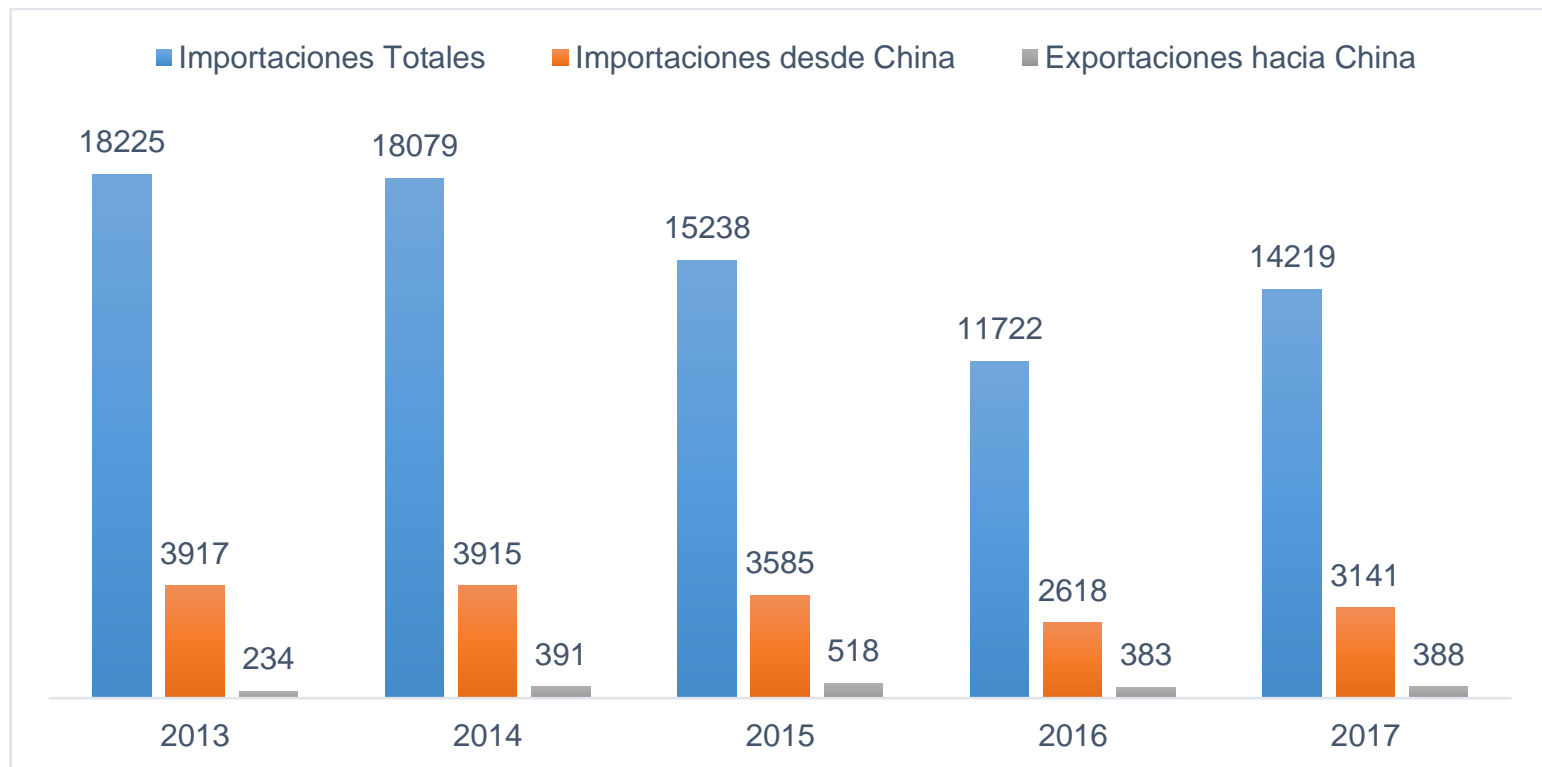

Figura 1 Diferencia entre las exportaciones e importaciones no petroleras entre Ecuador y China en millones de dólares Americanos (FOB)

Fuente: Ministerio de Comercio Exterior del Ecuador (2017)

Las importaciones que hizo Ecuador desde China en el 2013, 2014, 2015 han disminuido significativamente entre un año al otro; mientras que para el año 2016 estas disminuyeron considerablemente debido a la desaceleración económica en China, la caída de los precios del petróleo y otros productos básicos, las fuertes fluctuaciones de los tipos de cambio y la volatilidad financiera provocada por la divergencia de las políticas monetarias de los países desarrollados; además, a la aplicación de salvaguardias en el Ecuador desde el año 2015 hasta mayo del 2017; son algunos de los factores que influyeron en el comercio internacional (Examen Estadístico del Comercio Mundial 2016).

A pesar de haber disminuido, los porcentajes de los bienes importados desde China cada año se mantiene en un promedio de 3.639,50 millones; con excepción del año 2016 donde se observa una disminución del -26,97\% con relación al año 2015. 


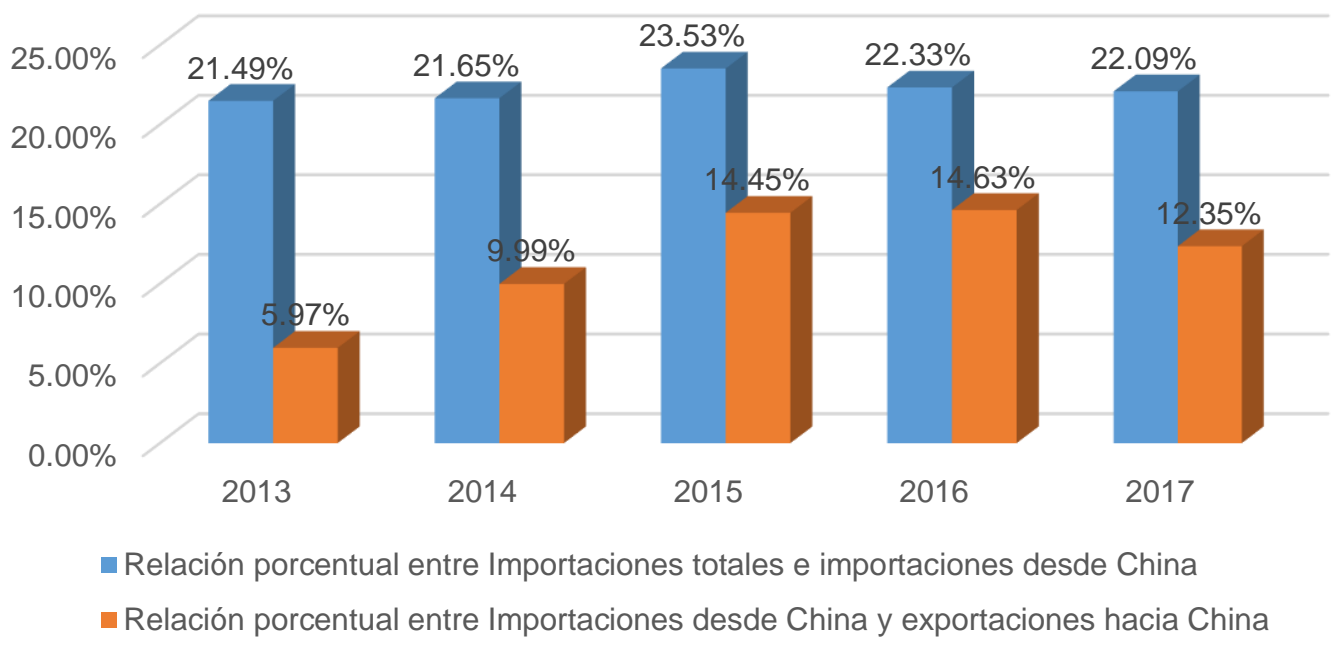

Figura 2 Diferencia porcentual entre importaciones totales e importaciones no petroleras y exportaciones no petroleras entre Ecuador y China (FOB)

Fuente: Ministerio de Comercio Exterior del Ecuador (2017)

El desarrollo significativo que ha experimentado China en estos últimos años, ha llevado a que entre en una franca expansión a nivel mundial; esto ha hecho que inicie una especie de "coloniaje" comercial en todos los mercados; del cual, el Ecuador no puede ser la excepción. Este pequeño país latinoamericano, se estableció como un proveedor de bienes en cantidades importantes; tanto es así que, del total de las importaciones no petroleras realizadas por el Ecuador desde el resto del mundo, aquellas provenientes de China representan entre el $21 \%$ y $23 \%$. En cambio, del total de las exportaciones al mercado internacional, las enviadas al mercado chino, fluctúan entre el $6 \%$ y $12 \%$.

De lo señalado brevemente en el párrafo anterior, se evidencia claramente un desbalance que obviamente perjudica en términos de la Balanza Comercial a la economía ecuatoriana.

\section{El Producto Interno Bruto}

Corresponde al valor monetario de la producción de bienes y servicios; además, refleja el estado de la economía de un país en un tiempo determinado (Terán, 2018). 


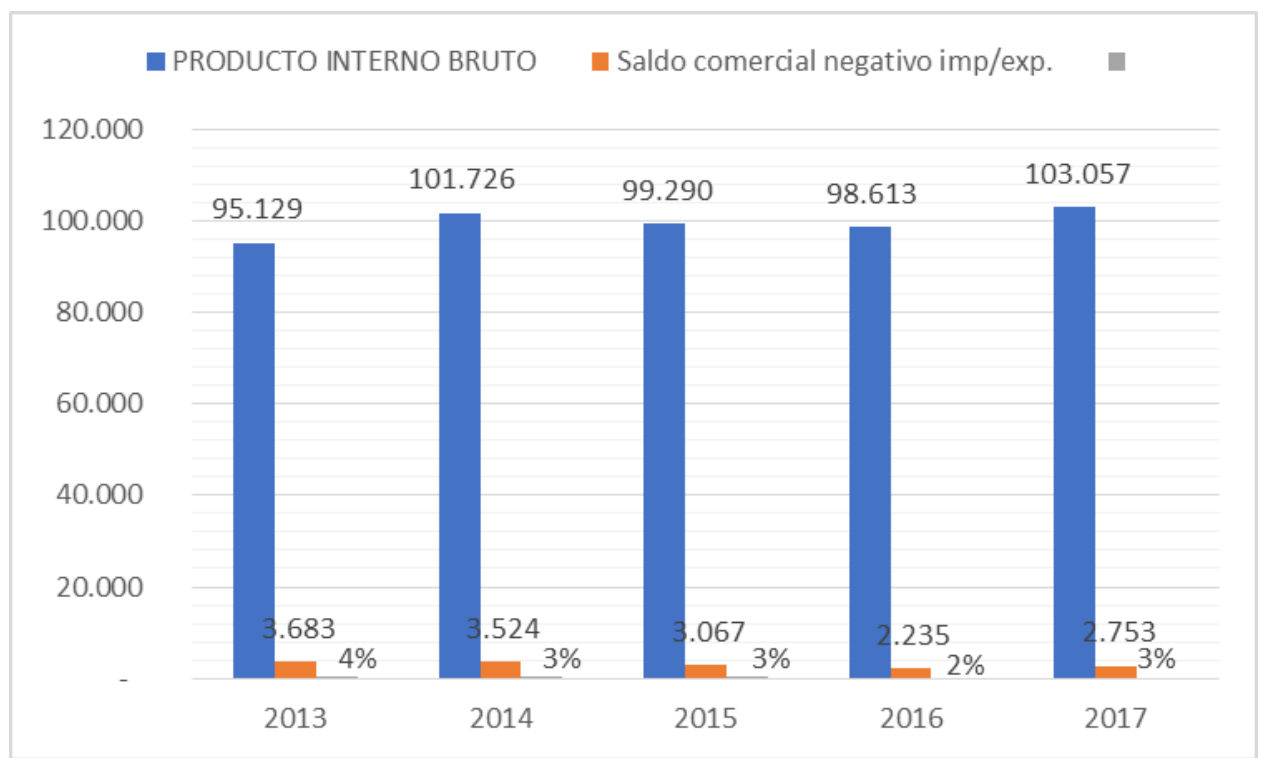

Figura 3 Diferencia porcentual entre el PIB y el saldo comercial negativo de importaciones no petroleras y exportaciones no petroleras entre Ecuador y China (FOB)

Fuente: Banco Central del Ecuador (2018)

Durante el período analizado el PIB mantuvo un comportamiento relativamente estable en términos absolutos, que oscila alrededor de los cien mil millones de dólares USA, con variaciones poco relevantes; así: los años 2014 y 2017 con 101.726 millones de dólares y 103.057 millones de dólares respectivamente.

Siendo la Balanza Comercial, un componente del Producto Interno Bruto, el desajuste comercial con China, obviamente influyó de manera negativa en su saldo; toda vez que el saldo comercial negativo correspondientes a las importaciones no petroleras y exportaciones no petroleras con China corresponden al 2\%, 3\% y $4 \%$ del PIB.

\section{Conclusiones}

Para Ecuador el impacto comercial con China; entre las importaciones no petroleras y exportaciones no petroleras han representado un saldo comercial negativo afectando al producto interno bruto (PIB) en un promedio del 3\% en los años analizados; por el contrario, para China una balanza comercial positiva.

El comercio entre Ecuador y China ha provocado una salida de capitales del USD 15.262 millones de dólares desde el 2013 al 2017, originados por el comercio internacional entre ambos países; ya que, una necesita de la otra para abastecerse de bienes y viceversa; logrando fortalecer y aumentar únicamente el comercio de China, más no el comercio del Ecuador.

La aplicación de aranceles (salvaguardias) para frenar las importaciones no petroleras en el Ecuador fue una estrategia acertada; ya que éstas se disminuyeron en el año 2016 donde se observa una disminución del -26,97\% con relación al año 2015. 
Esta competencia desigual entre el país asiático y Ecuador afecta al presupuesto general del Estado ya que la salida de recursos reduce los ingresos destinados para cubrir las obligaciones contraídas para la prestación de bienes y servicios públicos como: salud, educación, infraestructura, obra social entre otros.

Esto nos demuestra cuán dependiente es el Ecuador del gigante asiático ya que al incrementar las importaciones aumentan las exportaciones y viceversa. Con esta información comparativa se aprecia que el problema de la economía ecuatoriana radica en su matriz productiva, el país importa más de lo que produce, consume más de lo que vende, las exportaciones tienden a disminuir y sin salvaguardias las importaciones aumentan.

\section{Recomendaciones}

Es importante generar estrategias para aumentar las exportaciones no petroleras con China; exportando productos preferidos por el gigante asiático como: camarones, harina de pescado, banano entre otros productos consumidos, para equilibrar la balanza comercial entre ambos países, fortaleciendo no sólo la economía de China; sino también la economía del Ecuador.

Apoyar la producción y a la industrialización ayudaría a disminuir las importaciones y a aumentar las exportaciones hacia China, aunque es realmente imposible para Ecuador competir en el mercado internacional con el gigante asiático por las ventajas comparativas antes expuestas, lo más acertado sería buscar nuevos mercados o innovar en la producción.

\section{Bibliografía}

Banco Central del Ecuador. (2018). Cuentas Nacionales. Obtenido de https://www.bce.fin.ec/index.php/component/k2/item/763

Banco Central del Ecuador. (15 de Febrero de 2018). https://contenido.bce.fin.ec/documentos/Estadisticas/SectorExterno/BalanzaPagos/balanz aComercial/ebc201802.pdf.

Briones, R. A. (03 de 2018). La Política Comercial y las Importaciones de Bienes de Consumo en Ecuador. $\quad$ período 2010-2016. Obtenido de http://repositorio.ug.edu.ec/browse?type=author \&value=Briones+Mora\%2C + Raquel $+\mathrm{Ab}$ igail

Cámara de Comercio de Guayaquil. (2018). El Impacto de las Salvaguardias sobre la Poducción en el Ecuador. Departamento de Investigación y Proyectos. Recuperado el 21 de 11 de 2018 de http://www.lacamara.org/ccg/Docs\%20generales/E1\%20efecto\%20de\%20las\%20salvagu ardias\%20-\%20CCG.pdf

Cuentas Nacionales. (2018). Banco Central del Ecuador. Obtenido de https://www.bce.fin.ec/index.php/component/k2/item/763

De la Paz, M. (2018). Torniquete a la salida de dólares.

Durán, J., \& Pellandra, A. (2017). La Irrupción de China y su Impacto sobre la Estructura Productiva y Comercial en América Latina y el Caribe. Naciones Unidas, Santiago.: ISSN 1680-869X. 
Ellis, E. (2018). Hacia una Asociación Estratégica: Las Inversiones de China en América Latina. . Revista de Red CAEM., 27.

Examen Estadístico del Comercio Mundial 2016. (16 de 10 de 2019). El Comercio Mundial en 2015 - 2016.2 Obtenido de https://www.wto.org/spanish/res_s/statis_s/wts2016_s/WTO_Chapter_03_s.pdf

Feijoó, W. E. (04 de 2019). Impacto de las salvaguardias en la balanza de pagos en Ecuador, período 2015 - 2017. Universidad de Guayaquil, Facultad de Ciencias Económicas, Carrera de Economía. Guayaquil. Obtenido de http://repositorio.ug.edu.ec/bitstream/redug/40493/1/TFEIJOO\%20DURAZNO\%20WLADIMIR\%20ELIAS.pdf

Hernández, R., Fernández, C., \& Baptista, M. d. (2010). Metodología de la investigación, (ISBN: 978-607-15-0291-9) (Quinta. ed.). México.

Jiménez, H., Andrade, V., Sánchez, S., Bustos, P., Quishpe, J., \& Flores, S. (01 de Octubre de 2017). Ministerio de Comercio Exterior. Obtenido de https://www.comercioexterior.gob.ec/wp-content/uploads/2017/11/Bolet\%C3\%ADnMensual.pdf

La Hora. (18 de 07 de 2011). Comercio: Puntos Claves para Exportar a China. Obtenido de https://lahora.com.ec/noticia/1101174682/comercio-puntos-claves-para-exportar-a-china Mazo, E. (2014). China es ya la Primera Potencia Mundial. Expansión.com.

Ministerio de Comercio Exterior e Inversión. (Noviembre de 2017). Obtenido de https://www.comercioexterior.gob.ec/wp-content/uploads/2018/01/Informe-MensualDiciembre_4.pdf

Ministerio de Comercio Exterior e Inversión. (15 de 11 de 2018). Salvaguardia por Balanza de Pagos. Obtenido de https://www.comercioexterior.gob.ec/boletin-de-prensa-salvaguardiapor-balanza-de-pagos/

Ministerio de Comercio Exterior e Inversiones. Informe Mensual de Comercio Exterior. (Noviembre de 2017). Obtenido de https://www.comercioexterior.gob.ec/wpcontent/uploads/2018/01/Informe-Mensual-Diciembre_4.pdf

Mora, G. (Octubre de 2011). Las Importaciones de Calzado desde La República Popular China hacia el Ecuador Periodo 2009 - 2010. Universidad de Guayaquil. Facultad de Ciencias Económicas. Tesis para obtener el Título de Economista. Obtenido de http://repositorio.ug.edu.ec/bitstream/redug/2352/1/Mora\%20Orodo\%C3\%B1ez\%20Gen aro\%20Bolivar.pdf

Peña, H. (10 de Noviembre de 2018). Cámara de Comercio de Guayaquil. Relación Comercial Ecuador - China. Obtenido de http://www.lacamara.org/websiteantes/images/boletines/informacion-comercial/relacion\%20comercial\%20ecuador\%20$\% 20$ china.pdf

Peña, H. (15 de 10 de 2019). Relación Comercial ECUADOR - CHINA. Obtenido de http://www.lacamara.org/website-antes/images/boletines/informacioncomercial/relacion\%20comercial\%20ecuador\%20-\%20china.pdf

Perrotti, D. (2015). La República Popular de China y América Latina: Impacto del Crecimiento Económico Chino en las Exportaciones Latinoamericanas. Revista Cepal 116. Agosto 2015.

Ramírez, R. (2014). El "Sueño Chino" de XI JINPING. La "Quinta Generación de Dirigentes" y su Programa de Gobierno. (I. 2386-2491, Ed.) La Albolafia: Revista de Huamnidades y Cultura. 
Revista Líderes. (10 de 11 de 2018). Obtenido de https://www.revistalideres.ec/lideres/productosecuatorianos-demanda-china-mariella.html

Terán, N. C. (Julio de 2018). Análisis de los Efectos del Comercio Bilateral Ecuador - China en los Indicadores Macroeconómicos PIB, Balanza Comercial y Empleo del Ecuador, durante el perìodo 2013-2017. Quito.

Valencia, D. (2015). Ministerio de Comercio Exterior. Obtenido de https://www.comercioexterior.gob.ec/wp-content/uploads/downloads/2016/04/Informede-Gestion-Institucional-MCE-2015.pdf

Zurita, A. (4 de Marzo de 2016). Instituto Español de Estudios Estratégicos. China: El Despertar del Dragon Rojo. Obtenido de http://www.ieee.es/Galerias/fichero/docs_marco/2016/DIEEEM032016_China_Despertar_DragonRojo_AlfonsoZurita.pdf 